\title{
Automatización de procesos industriales mediante Industria 4.0
}

DOI: https://doi.org/10.33262/ap.v3i3.1.80

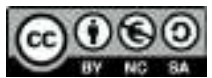

Automation of industrial processes through Industry 4.0

Gustavo Barona López. ${ }^{1}$ \& Luis Efraín Velasteguí. ${ }^{2}$

\begin{abstract}
The paper aims to present the automation of industrial processes through Industry 4.0, to establish hierarchical levels of automation from the automation pyramid. For this, a theory about Industry 4.0 was structured in relation to the automation pyramid. Subsequently, the automation of industrial processes about beverage packaging, flow measurement, wireless network technology, total integration of levels and oil refining is presented, in each one its automation architecture is shown to perform the evaluation of its reference levels to the automation pyramid concept. In the evaluation, the field instruments, control and SCADA system were described, in relation to the automation pyramid. Subsequently, the automation of industrial processes of beverage packaging, flow measurement, wireless network technology, total integration of levels and oil refining is presented, in each one its automation architecture is shown to carry out the evaluation of its levels in reference to the concept of the automation pyramid. In the evaluation, the field instruments, control and SCADA system were described in relation to the automation pyramid. Concluding that the Industrial Ethernet communication network, due to its high capacity to transmit data between company levels, long cabling, ease of communication between controllers and ideal for local area networks (LAN), is used at Level 2, Level 3 and Level 4, while PROFIBUS, PROFINET, HART, IO-Link and AS-Interface communication networks, for their real-time data transfer between field instruments and controllers, work in explosive environments, data traffic handling, data
\end{abstract}

1 Escuela Politécnica Nacional, Facultad de Ingeniería Mecánica. Quito, Ecuador, barona_gustavo@hotmail.com

${ }^{2}$ Editorial Ciencia Digital. Ambato, Ecuador, luisefrainvelastegui@cienciadigital.org 
recovery on the network, etc., is used in Level 0 and Level 1 in the automation of industrial processes.

Keywords: Industrial automation 4.0, Industry 4.0, automation of industrial processes, automation pyramid, industrial communication networks.

\section{Resumen}

El articulo tiene por objetivo presentar la automatización de procesos industriales mediante Industria 4.0, para establecer los niveles jerárquicos de automatización a partir de la pirámide de automatización. Para esto, se estructuró una teoría acerca de Industria 4.0 con relación a la pirámide de automatización. Posteriormente se presenta la automatización de procesos industriales relacionados a envasado de bebidas, medición de flujo, tecnología de red Wireless, integración total de niveles y refinación de petróleo, en cada uno se muestra su arquitectura de automatización para realizar la evaluación de sus niveles en referencia al concepto de pirámide de automatización. En la evaluación se describieron los instrumentos de campo, control y sistema SCADA, en relación a la pirámide de automatización. Se concluye que la red de comunicación Ethernet industrial por su alta capacidad de transmitir datos entre los niveles de empresa, cableado de gran extensión, facilidad de comunicación entre controladores e ideal para redes de área local (LAN), es utilizada en el Nivel 2, Nivel 3 y Nivel 4, mientras que las redes de comunicación PROFIBUS, PROFINET, HART, IO-Link y AS-Interface, por su transferencia de datos en tiempo real entre instrumentos de campo y controladores, trabajo en ambientes explosivos, manejo de tráfico de datos, recuperación de datos en la red, entre otras, es utilizada en el Nivel 0 y Nivel 1 en la automatización de procesos industriales.

Palabras claves: Automatización industrial 4.0, Industria 4.0, automatización de procesos industriales, pirámide de automatización, redes de comunicación industrial.

\section{Introducción}

La transformación de materias primas en productos manufacturados tiene un ritmo acelerado debido al crecimiento y la demanda de la población mundial, lo que ha originado una nueva revolución industrial denominada Industria 4.0 que tuvo su nacimiento en el año 2011 en el país de Alemania. Este nuevo modelo de industria requiere que la instrumentación, control, supervisión, fábrica y empresa, estén interconectados mediante una jerarquización de niveles que proporcionen procesos de automatización eficientes y de bajo costo de instalación, con el fin de satisfacer la demanda de productos para que las empresas puedan competir en el sector industrial. Esta jerarquización se representa en la denominada pirámide de automatización que consta de cinco niveles de automatización en la empresa.

El flujo de información entre los niveles de la pirámide de automatización se lo realiza mediante redes de comunicación industrial que utilizan un simple cable común para los 
dispositivos a ser conectados lo que permite tener una periferia descentralizada en cada nivel de la pirámide, lo cual representa una solución respecto al extenso cableado punto a punto que es de tipo centralizado y que aún en la industria ecuatoriana se lo utiliza en la automatización de sus procesos industriales.

En la presente investigación se ha realizado para varios procesos industriales la evaluación de los cinco niveles jerárquicos de la pirámide de automatización en un entorno de Industria 4.0. La evaluación muestra que el desarrollo de las redes de comunicación industrial son la tecnología que permite la integración de los instrumentos industriales de forma estructurada y jerárquica en los niveles de la pirámide.

\section{Industria 4.0}

A continuación, se presentan conceptos de la Industria 4.0 con referencia a la pirámide de automatización, la cual consta de cinco niveles jerárquicos, esto con el fin de desarrollar una teoría que permita describir los niveles de la pirámide de automatización en los casos de automatización de procesos industriales que serán presentados.

\section{Concepto de Industria 4.0}

Industria 4.0 es la cuarta revolución industrial que logra la interconexión de los sistemas productivos industriales con la sociedad digital para satisfacer las demandas de consumo de las personas, a un nivel acelerado de extracción y procesamiento de las materias primas de la tierra, utilizando tecnologías como big data, comunicación de la nube, robótica avanzada, inteligencia artificial, blockchain, internet de las cosas, internet del todo, computación cuántica y en la nube, impresión 3D, sistemas ciberfísicos, ciberseguridad, automatización industrial Wireless, redes industriales, redes cognitivas, virtualización de redes, 6G, comunicación molecular, comunicaciones verdes, realidad aumentada, nanotecnología, TICs, entre otras, todo esto mediante estrategias innovadoras, creativas y eficientes que logren minimizar el impacto ambiental en beneficio de la sostenibilidad económica y ambiental del planeta.

\section{Automatización industrial mediante industria 4.0}

Toda empresa industrial busca la eficiencia económica y productiva para ser sostenible en el mundo actual, lo que ha originado que los procesos de manufactura evolucionen a la denominada cuarta revolución industrial, llamada Industria 4.0. Esta Industria 4.0 requiere la conexión de la instrumentación industrial con las áreas de la empresa mediante niveles jerárquicos que permiten su automatización, para esto, la Industria 4.0 ha establecido el concepto de pirámide de automatización (Witorg, 2019) (ARC Advisory Group, 2015).

\section{Proceso para la automatización industrial mediante industria 4.0}

En la actualidad, la automatización de las compañías, empresas, transnacionales, entre otras, para fabricar un producto manufacturado, se está realizando bajo el sistema de arquitectura denominado Industria 4.0. En este sistema el proceso de automatización 
industrial se basa en la pirámide de automatización que generalmente consta de 5 niveles, o en otros casos más debido a que la Industria 4.0 va evolucionando al ritmo de la rápida transformación de materias primas en productos de ingeniería. Una de las diferencias de la tradicional automatización con la pirámide, es que, en esta última la conexión de sensores, actuadores, controladores, SCADA y todo el nivel corporativo, es mediante redes de comunicación industriales.

\section{Pirámide de automatización}

La pirámide de automatización es una representación gráfica jerarquizada de los niveles de automatización industrial que requiere la industria para su funcionamiento, acorde a las nuevas tecnologías de la comunicación en un entorno de Arquitectura Industrial 4.0. Esta tendencia que se viene dando años atrás garantiza la efectividad productiva, administrativa y económica de las compañías, empresas, transnacionales, entre otras.

Si bien es cierto que el concepto de pirámide de automatización tuvo sus comienzos en el año de 1973, con la publicación del libro Computer Integrated Manufacturing por parte de Joseph Harrington, no fue hasta el año de 1984 que las industrias comenzaron a ver su potencial beneficio. El termino Computer Integrated Manufacturing (CIM), que en español significa Manufactura Integrada por Computador, es un concepto muy amplio puesto que reúne un gran número de tecnologías y conceptos como la informática, redes industriales, diseño asistido por computador, pirámide de automatización, entre otras. Esta pirámide de automatización también es conocida como pirámide CIM y en la actualidad es incorporada a los conceptos de Industria 4.0 para mejorar la automatización de procesos industriales. (Salvendy, 2001) (Higuera y Castillo, 2007).

La pirámide de automatización generalmente tiene 5 niveles estructurados estratégicamente para su intercomunicación en el proceso productivo. Esta pirámide sirve para la correcta estructuración del proceso de automatización en la planta industrial, por tal motivo, para tener un conocimiento a nivel de gerencia se debe tener presente el objetivo de cada nivel (Barrientos y Gambao, 2014) (García, 2001).

La Figura 1 muestra la pirámide de automatización con sus respectivos niveles jerárquicos. La jerarquización hace posible determinar la clase de instrumentos o herramientas tecnológicas que se usan en cada piso de la pirámide. El Nivel 0 contiene sensores, actuadores y todo dispositivo de campo. En el Nivel 1 se encuentran controladores lógicos programables (PLC), sistemas de control distribuido (DCS), sistemas de control numérico, tarjetas basadas en microprocesadores o microcontroladores, computadores industriales, entre otros. El Nivel 2 lo conforma el sistema de Supervisión, Control y Adquisición de Datos (SCADA). En el Nivel 3 está instalado el Sistemas de ejecución de fabricación (MES). En el Nivel 4 se tiene el sistema de Planificación de Recursos Empresariales ERP (Witorg, 2019) (Barrientos y Gambao, 2014) (García, 2001). 


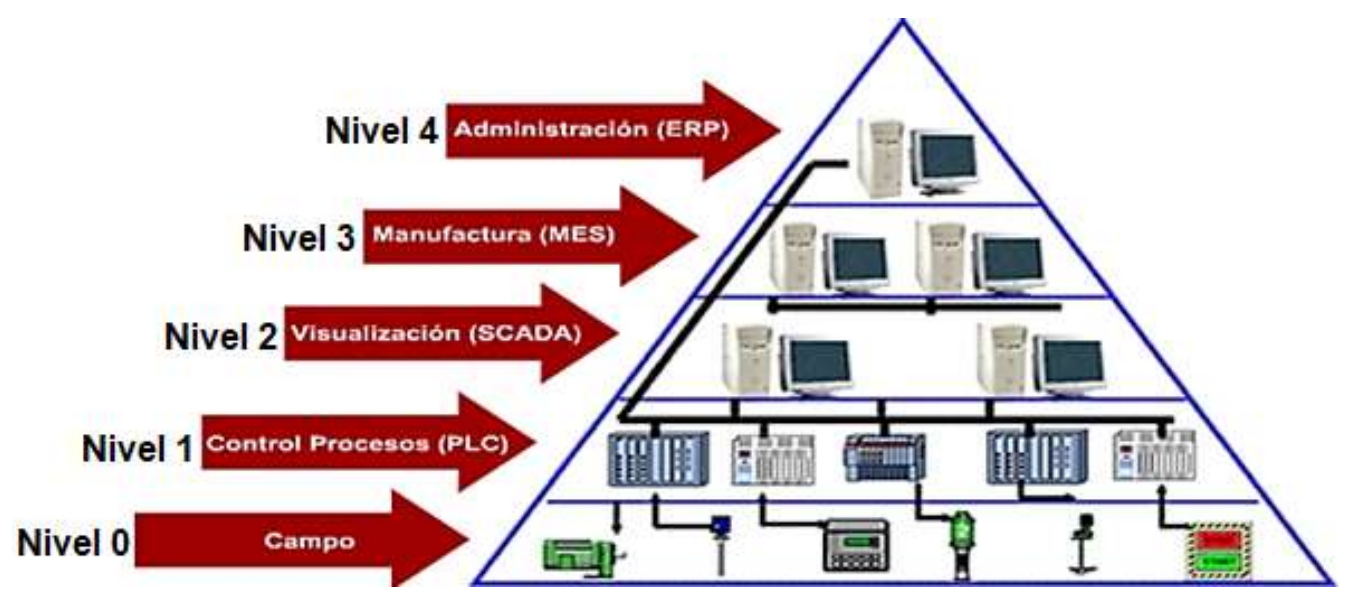

Figura 1. Pirámide de automatización (Lozano y Zamora, 2008)

Los sistemas de producción evolucionan al ritmo de los nuevos problemas de sostenibilidad del mundo, con lo cual, la Industria 4.0 definió un nuevo concepto de arquitectura RAMI 4.0 (Reference Architectural Model Industry 4.0) y lo integró con los 5 niveles de la pirámide de automatización, haciendo que la misma tenga 7 niveles, como se muestra en la Figura 2, de esta forma se logró adaptar producto-industria al mundo conectado (Tecnológico Monterrey, 2015). Este nuevo concepto contiene:

Los cinco niveles de la pirámide de la automatización, pero agrega dos niveles más: el "Producto" en la parte inferior de la pirámide, y el "Mundo Conectado" en la parte superior de la pirámide como se muestra en la Figura 2. Integra y adopta todas las definiciones y modelos de referencia de las normas IEC 62264 y IEC 61512. (Tecnológico Monterrey, 2015, pág. 4)

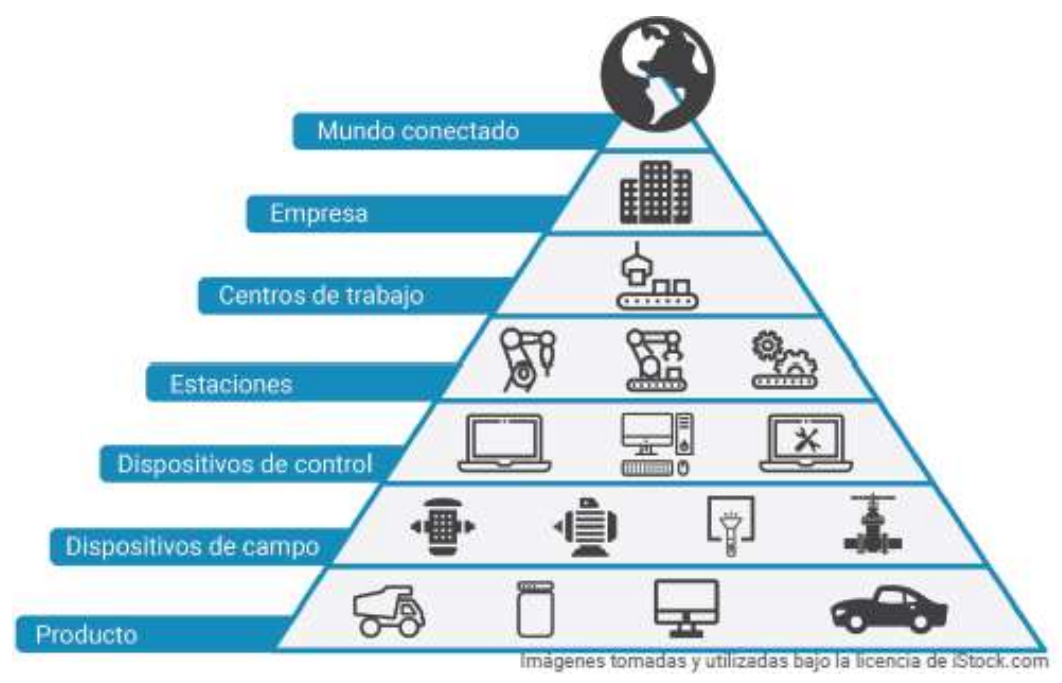

Figura 2. Concepto RAMI añadido a los 5 niveles de la pirámide de automatización (Tecnológico Monterrey, 2015)

\section{Niveles de la pirámide de automatización.}

Para estructurar los niveles de automatización en empresas se utilizada la pirámide de automatización que consta de 5 niveles jerárquicos que son detallados a continuación. 


\section{Nivel 0 nivel de procesos.}

Es el nivel más bajo en la jerarquía de la pirámide de automatización, donde se encuentran sensores, actuadores y todo dispositivo de campo. El Nivel 0 se podría decir que son las piezas que conforman una máquina. Realizando una analogía con el cuerpo humano se puede decir que los sensores son los nervios y los actuadores son los músculos. Los sensores y actuadores que interactúan directamente con el proceso productivo suelen recibir el nombre de dispositivos de campo (field devices) (Barrientos y Gambao, 2014) (García, 2001).

De forma general, los sensores son dispositivos que captan las señales analógicas y/o digitales del proceso industrial y envían esta información a un controlador (cerebro electrónico). El controlador evalúa, compara y procesa la información, con el fin de emitir una señal de salida que llegan a los actuadores, los mismos que accionan los mecanismos de la maquinaria para cumplir el proceso industrial (Barrientos y Gambao, 2014) (García, 2001).

\section{Nivel 1 nivel de control}

En el Nivel 1 se controlan las máquinas que intervienen en el proceso de producción. El control de movimientos de las máquinas se lo realiza mediante un cerebro inteligente programable, el mismo que emite señales que son ejecutadas por los actuadores. El cerebro inteligente se lo denomina controlador y son del tipo controladores lógicos programables (PLC), controladores de automatización programable (PAC), sistemas de control distribuido (DCS), sistemas de control numérico, tarjetas basadas en microprocesador o microcontroladores, computadores industriales, entre otros (Barrientos y Gambao, 2014) (García, 2001).

Este nivel recibe información del Nivel 0 y envía órdenes para que los elementos industriales ejecuten sus funciones. También este nivel se comunica con el nivel 2 que es el de supervisión del proceso industrial (Witorg,2019).

\section{Nivel 2 nivel de supervisión (SCADA)}

En el Nivel 2 se encuentra el sistema de Supervisión, Control y Adquisición de Datos (SCADA) que está integrado por hardware, software y red de comunicación. En este nivel se observa el proceso productivo en forma gráfica y animada, a través de la interfaz hombre maquina (HMI). Por medio de la HMI se permite la gestión y control de cualquier sistema local o remoto gracias a una interfaz gráfica que comunica al usuario con el sistema (Witorg, 2019) (Pérez, 2015).

El hardware es la unidad terminal maestra (MTU) que puede ser una unidad central de procesamiento (CPU) o un PLC maestro, su objetivo es la de supervisar y evaluar la información, además de, recibir y emitir señales para su funcionamiento y control. Esta MTU recopila información del Nivel 0 como del Nivel 1 donde se encuentran subestaciones conocidas como unidades terminales remotas (RTU) que son del tipo PLC 
(conexión esclavo o servidor), microprocesadores, tarjetas electrónicas, etc. (Pérez, 2015).

El software permite la interacción hombre-máquina, a través de su instalación en la MTU que está conectada a un monitor, en el cual, se observan las variables físicas del proceso productivo como presión, temperatura, caudal, entre otras. Esta interacción da como resultado que el jefe de producción pueda interpretar de forma numérica, gráfica y animada, la información que se tiene el Nivel 0 , con el fin poder ejecutar comandos hacia las unidades terminales remotas del Nivel 1, y estos a su vez controlar sensores, actuadores y otros dispositivos de campo, para el control de las máquinas (Witorg, 2019) (Pérez, 2015).

\section{Nivel 3 nivel de fábrica}

El Nivel de fábrica es denominado nivel de planificación o nivel de sistemas de ejecución de fabricación (MES) y tiene que ver con las operaciones realizadas y monitorización de la planta industrial. En este sistema se realizan las tareas de programación de la producción, gestión de materiales, gestión de compras, análisis de costos de fabricación, control de inventarios, gestión de recursos de fabricación, gestión de calidad, gestión de mantenimiento, esto permite a la gerencia ver lo que está sucediendo y tomar decisiones basadas en esa información (Witorg, 2019) (Barrientos y Gambao, 2014) (García, 2001)

El Nivel de fábrica emite los programas hacia el nivel 2 y recibe de este las incidencias de la planta en minutos, horas, turnos o incluso días, por tal motivo su capacidad de almacenamiento es menos exigente. Este nivel suele utilizar un sistema de gestión informática por eso es conocido como MES (Witorg, 2019) (García, 2001).

\section{Nivel 4 nivel de empresa}

El Nivel de empresa (nivel corporativo o nivel administrativo) es el nivel más alto de la pirámide de automatización, por lo tanto, gestiona e integra todos los niveles anteriores. Para su gestión utiliza un sistema de Planificación de Recursos Empresariales ERP (Enterprise Resource Planning). El Nivel 4 y el Nivel 3 en algunas organizaciones están englobados como un mismo sistema (Witorg, 2019) (Barrientos y Gambao, 2014).

En el Nivel de empresa se realizan las tareas de gestión comercial y marketing, planificación estratégica, planificación financiera y administrativa, gestión de recursos humanos, ingeniería de producto, ingeniería de proceso, gestión de tecnología, gestión de sistemas de información, investigación y desarrollo (García, 2001).

\section{Redes de comunicación industrial}

Las redes de comunicación industrial permiten la transferencia de datos entre los diferentes niveles de la pirámide de automatización las cuales pueden ser divididas en redes de datos o información que están ligada a la parte superior de la pirámide y las redes de control o buses de campo (field bus) que están ligadas a la parte inferior de la pirámide 
(Prado, 2010) (Caler, 2015). En la Figura 3 se muestra el posicionamiento de las redes de comunicación industrial en la pirámide de automatización.

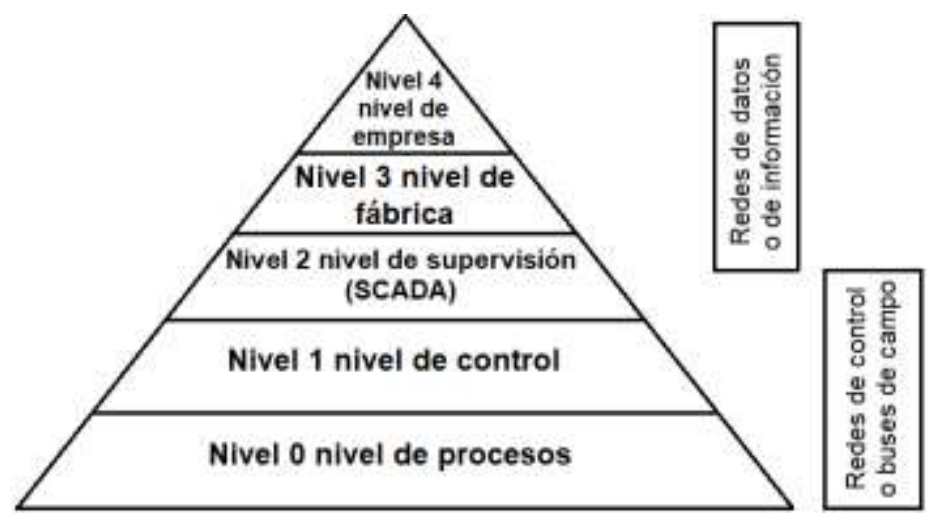

Figura 3. Posicionamiento de las redes de comunicación industrial en la pirámide de automatización

Fuente: Elaboración propia

La transmisión de datos entre los niveles de la pirámide automatización se transfiere mediante redes de comunicación y su selección para ser instaladas en cada nivel va de acuerdo al tipo, volumen y velocidad de transferencia de datos; el ambiente de trabajo, interconectividad entre instrumentos industriales; conexión de dispositivos de fabricación como robots, máquinas de control numérico; manejo de tráfico de datos, entre otras. En la Figura 4 se muestra los algunos tipos de redes de comunicación industrial que son utilizados para la conexión entre los niveles jerárquicos de la automatización.

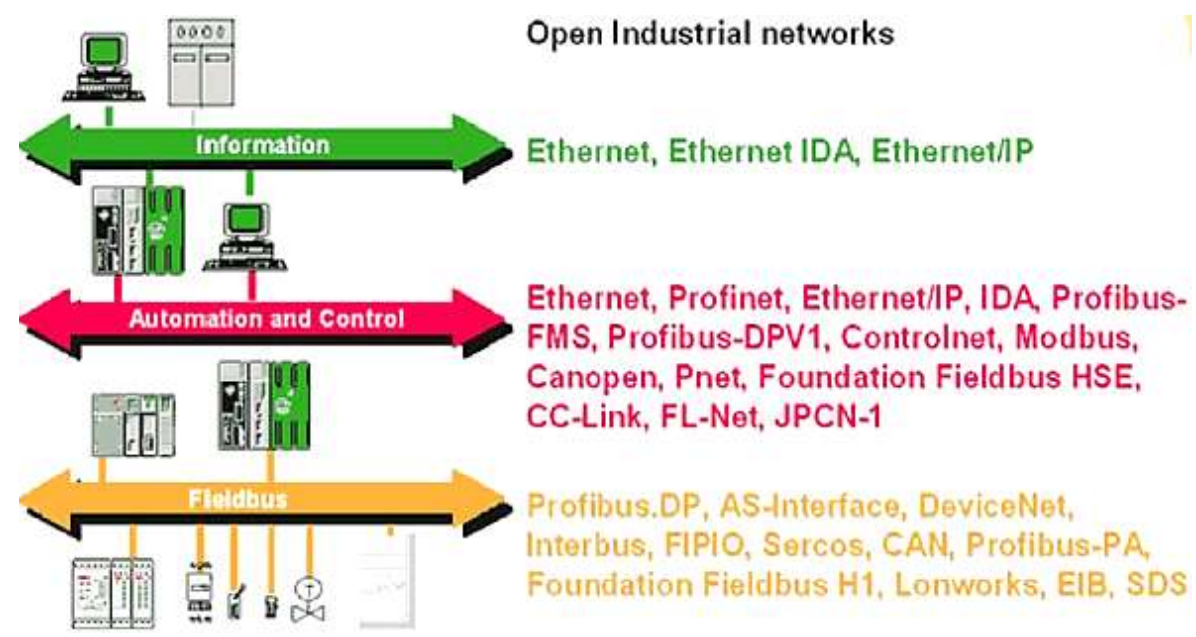

Figura 4. Tipos de redes de comunicación industrial (Caler, 2015)

Actualmente el bus de campo está reemplazando a las conexiones punto a punto que son sistemas de control aislados y centralizados con un funcionamiento de bucle de corriente de 4 a 20 mA, la tecnología de bus de campo es la red de comunicación más sofisticada, ya que facilita el control distribuido entre dispositivos de campo y controladores. (Salazar y Correa, 2011) (Universidad de Valencia, 2009). 
El objetivo de los buses de campo es reemplazar los sistemas de control centralizados que utilizan un extenso cableado convencional punto a punto por redes de control distribuido que están constituidos generalmente de fibra óptica. Estos buses de campo que poseen control distribuido reducen costos y mejoran la transmisión de datos ya que es digital (Prado, 2010) (Hurtado, 2015). En la Figura 5 se muestra la diferencia entre el tradicional sistema de cableado convencional versus la red de comunicación mediante bus de campo.
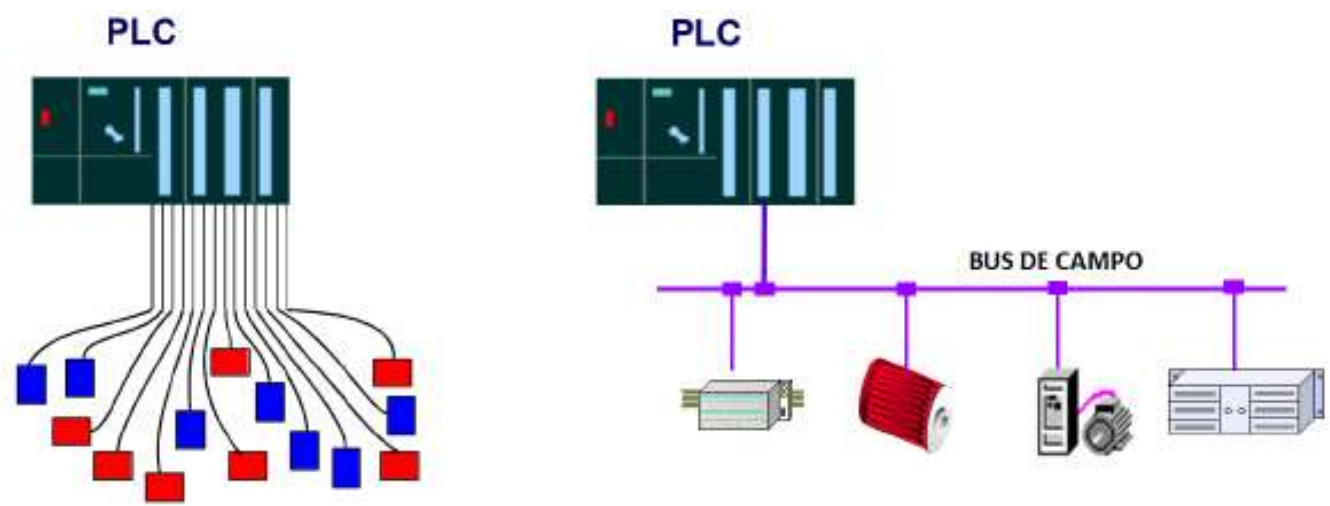

Figura 5. Sistema de cableado versus bus de campo (Hurtado, 2015)

\section{Automatización de procesos industriales mediante industria 4.0}

Se presenta la automatización de procesos industriales relacionados a envasado de bebidas, medición de flujo, tecnología de red Wireless, integración total de niveles y refinación de petróleo, en cada uno se muestra su arquitectura de automatización para evaluar en referencia al concepto de pirámide de automatización sus niveles jerárquicos. Como parte de un análisis se presentan resultados obtenidos.

\section{Automatización de un proceso de envasado de bebidas}

La Figura 6 es la arquitectura de automatización de un proceso de envasado y embotellado de bebidas, en donde se muestra el Nivel 0 y Nivel 1 de la pirámide de automatización. En toda la planta se utiliza la red de comunicación PROFINET, permitiendo una red troncal óptima y flexible que está conectada al Nivel 3 por medio de un switch de gestión (managed switch), su alto ancho de banda permite soluciones de control de movimiento eficientes y flexibles, además, por las características de esta red, se conectan motores, bombas y medidores de flujo de alta tecnología. En cambio, la red de comunicación PROFIBUS PA se acopla a la red PROFINET mediante un acoplador de segmentos SK3 Power Hub con tecnología Proxy. Mediante la red PROFIBUS PA se conectan sensores, transmisores de presión, nivel, temperatura y posición, en zonas con atmosferas explosivas. (Profibus, 2019). 


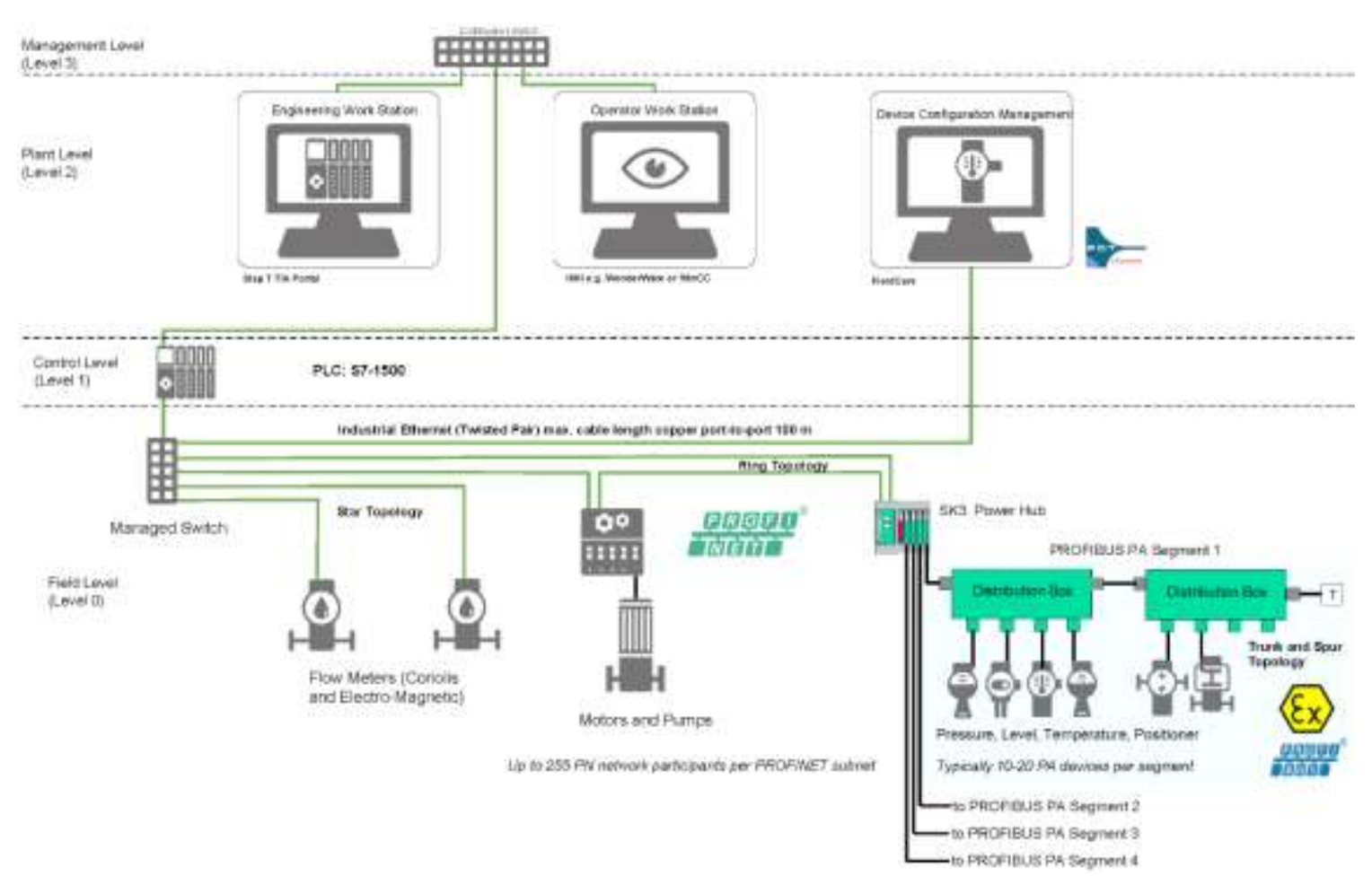

Figura 6. Arquitectura de automatización de un proceso de envasado y embotellado de bebidas (Profibus, 2019)

La Figura 6 muestra el Nivel 2 que es el nivel de Supervisión, Control y Adquisición de Datos (SCADA) que consta de la estación de trabajo de ingeniería (Engineering Work Station), la estación de trabajo del operador (Operator Wor Station) y la dirección de la configuración de dispositivos (Device Configuration Management), en los cuales están instalados los programas Step 7 Tia Portal, WonderWare y FieldCare respectivamente. En el monitor de cada estación se da la interfaz HMI que permite el control del proceso productivo (Profibus, 2019).

\section{Automatización de un proceso de medición de flujo}

La Figura 7 muestra la arquitectura de automatización de un proceso de medición dinámica de flujo, el cual puede ser utilizado para la medición del caudal de lácteos o bebidas en la industria alimenticia, lodos y residuos en la industria minera, hidrocarburos en la industria petrolera, entre otras. Las redes industriales que se ven en la Figura 7 delimitan el Nivel 0, Nivel 1 y Nivel 2 de la pirámide de automatización. 


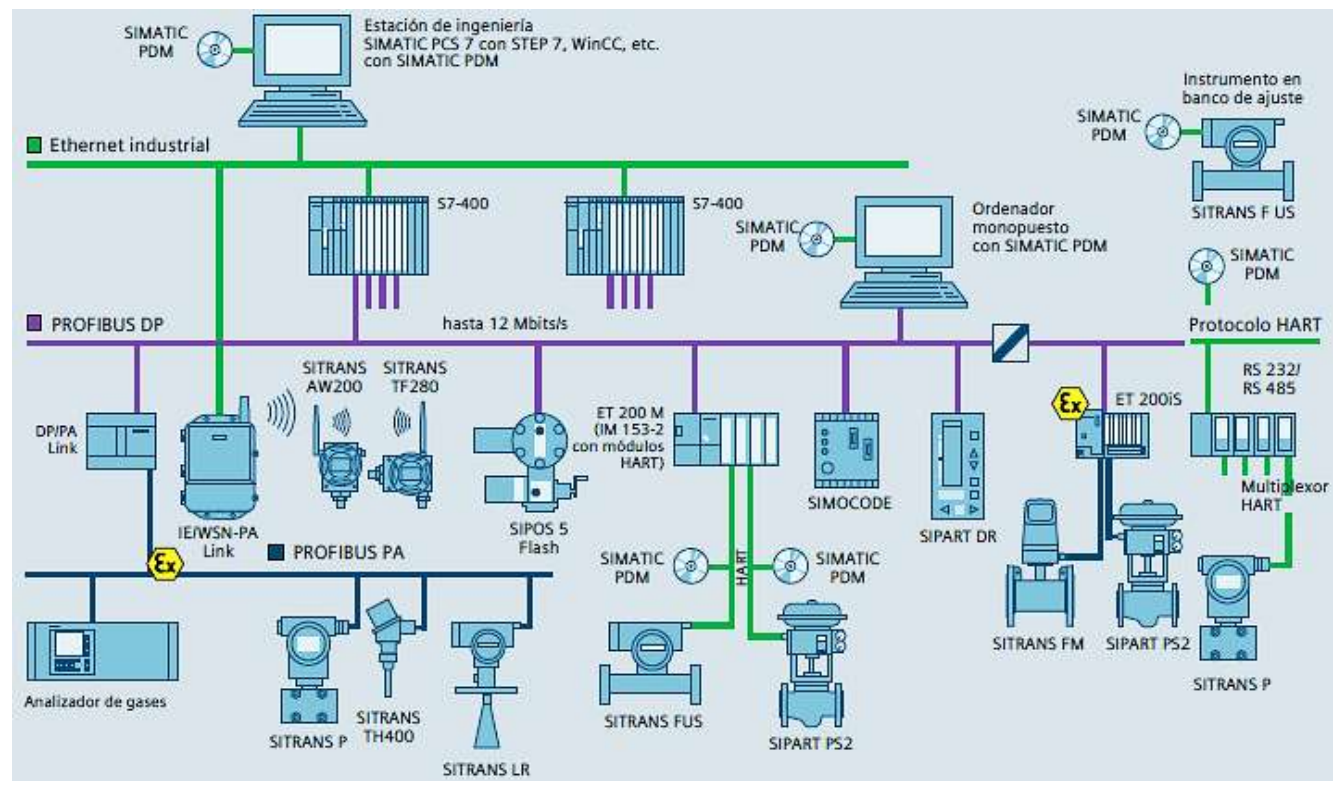

Figura 7. Arquitectura de automatización de un proceso industrial para la medición dinámica de flujo (Siemens, 2016)

En el Nivel 0 se encuentran instrumentos de campo como un transmisor de presión SITRANS P, válvula de control SIPART PS2 y medidor de flujo SITRANS FUS, que utilizan una red de comunicación HART; válvula de control SIPART PS2, medidor de flujo SITRANS FM, medidor de nivel por radar SITRANS LR, transmisor de temperatura SITRANS TH400, transmisor de presión SITRANS P y un analizador de gases, que utilizan una red de comunicación PROFIBUS PA. Como parte del Nivel 0 se tiene un adaptador de campo Wireless SITRANS AW200 que se acopla a los aparatos de campo y un transmisor de temperatura Wireless SITRANS TF280 para la entrada y salida de datos vía inalámbrica, los mismos que utilizan una red de comunicación Wireless HART.

En el Nivel 1 se encentra el PLC S7-400 que se conecta con el Nivel 0 mediante acopladores DP/PA Link, ET 200 M, ET 200iS y Multiplexor HART. En el Nivel 1 también se tienen controladores que son controlados por el PLC S7-400, estos son el controlador de procesos SIMOCODE para control de motores y SIPART DR para control de procesos. Como parte del Nivel 1 se tiene una pasarela IE/WSN-PA Link que conecta la red Wireless HART con la red Ethernet. La red de comunicación de datos PROFIBUS DP permite establecer la conectividad del Nivel 1 con el Nivel 0.

En el nivel 2 se encuentra el sistema de Supervisión, Control y Adquisición de Datos (SCADA), que está integrado por hardware, software y red de comunicación. El hardware es una estación de ingeniería SIMATIC PCS 7 y un ordenador monopuesto, en los cuales se ha instalado el software SIMATIC PDM. La red de comunicación de datos Ethernet industrial permite al sistema SCADA establecer conectividad directa con los controladores del Nivel 1 y conectividad indirecta con los instrumentos de campo del Nivel 0. 


\section{Automatización de un proceso con tecnología de red Wireless}

La Figura 8 muestra la arquitectura de automatización de un proceso industrial con tecnología de red Wireless (inalámbrica). A pesar que, esta tecnología tiene un suministro continuo de energía debido a la utilización de baterías internas puede existir momentánea perdida de señal e interferencias a causas de otras señales, su implementación como complemento en procesos industriales ofrece ciertas ventajas en comparación a las redes de comunicación mediante cableado. A partir de la Figura 8 se puede establecer el Nivel 0, Nivel 1 y Nivel 2 de la pirámide de automatización.

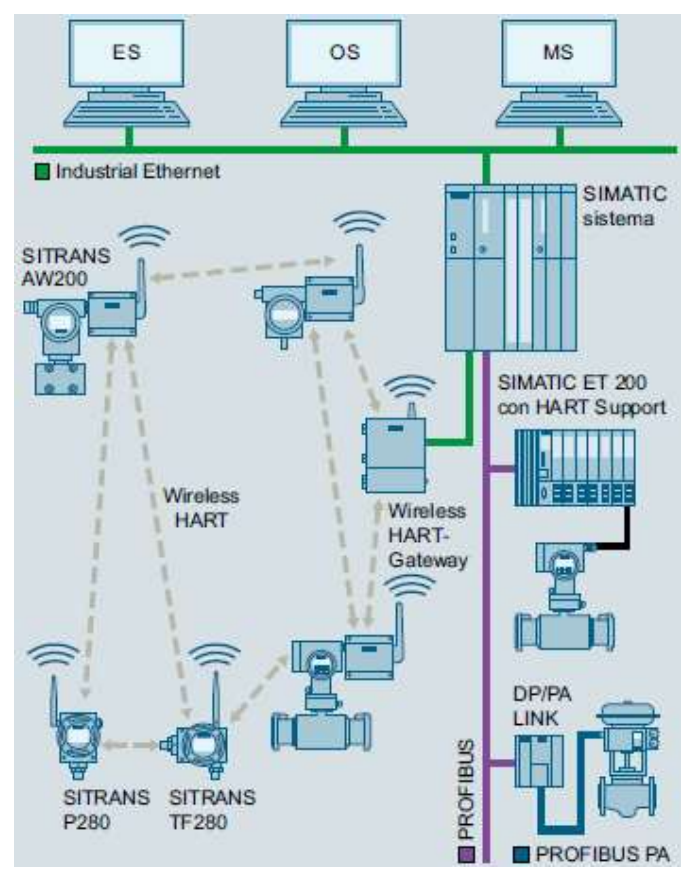

Figura 8. Arquitectura de automatización de un proceso con tecnología Wireless (Siemens, 2021)

En el Nivel 0 se encuentran los instrumentos de campo como la válvula de control y medidor de flujo que utilizan una red de comunicación PROFIBUS; transmisor de temperatura Wireless SITRANS TF280, transmisor de presión Wireless SITRANS TF280 y dos adaptadores de campo Wireless SITRANS AW200 que utilizan una red de comunicación WirelessHART.

En el Nivel 1 se encentra el controlador SIMATIC sistema que se conecta con el Nivel 0 mediante los acopladores SIMATIC ET 200 y Gateway. Las redes de comunicación de datos WirelessHART y PROFIBUS permiten la conexión del Nivel 1 con el Nivel 0.

En el nivel 2 se encuentra el sistema de Supervisión, Control y Adquisición de Datos (SCADA), que está integrado por hardware, software y red de comunicación. El hardware está integrado por tres unidades de procesamiento que son la estación de ingeniería (ES), la estación de operación (OS) y la estación de mantenimiento (MS), en los cuales es instalado generalmente el software SIMATIC PDM. La red de comunicación de datos 
Ethernet industrial permite al sistema SCADA establecer conectividad directa con los controladores del Nivel 1 y conectividad indirecta con los instrumentos de campo del Nivel 0.

\section{Automatización de un proceso con integración total de niveles}

La Figura 9 muestra la arquitectura de automatización de un proceso industrial mediante los cinco niveles jerárquicos de la pirámide de automatización, bajo un entorno de Arquitectura Industrial 4.0, ya que se gestiona la instrumentación industrial, los sistemas de ejecución para manufactura y la administración corporativa de la empresa. En la Figura 9 se puede ver como los instrumentos de campo interactúan con los instrumentos de control, y estos su vez interactúan con las diversas unidades terminales maestras, y todo integrado con el sistema de ejecución de fabricación (MES) y con la Planificación de Recursos Empresariales (ERP), además se observa las redes de comunicación en los diferentes niveles de automatización.

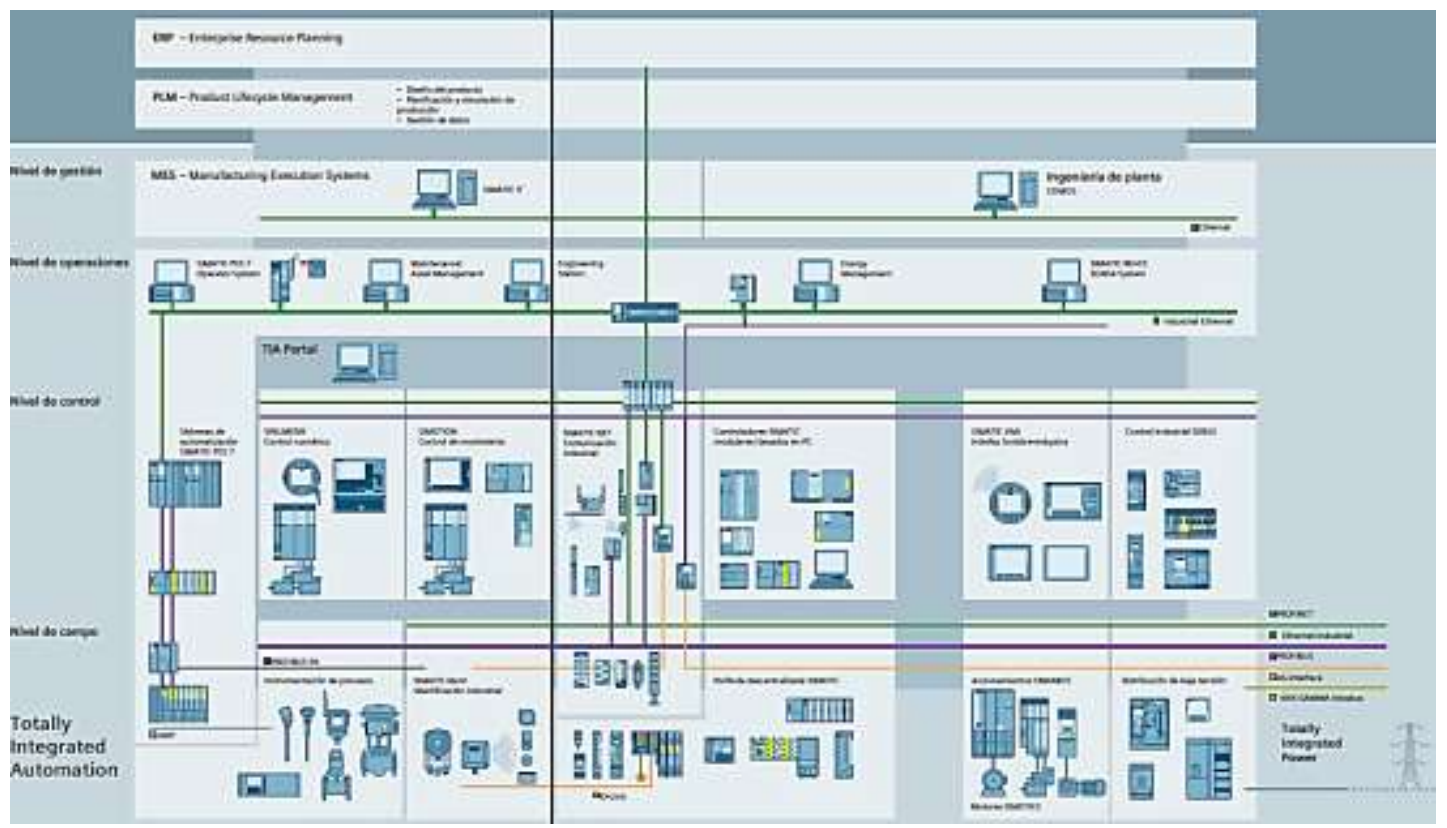

Figura 9. Arquitectura de automatización de un proceso industrial mediante los cinco niveles de la pirámide de automatización (Siemens, 2016)

En la Figura 9 se muestran todos los niveles de la pirámide de automatización, los mismos que se especificaron sus funciones en el ítem 2. A continuación se realizara una breve enumeración de los mismos.

El Nivel de campo corresponde al Nivel 0.

El Nivel de control es el Nivel 1.

El Nivel de operaciones o de supervisión (SCADA) pertenece al Nivel 2.

El Nivel de gestión llamado nivel de fábrica o nivel de MES corresponde al nivel 3.

El Nivel ERP o denominado Nivel de empresa pertenece al Nivel 4. 


\section{Automatización de un proceso de refinación de petróleo}

La Figura 10 muestra la arquitectura de automatización de un proceso de refinación de petróleo mediante los 5 niveles jerárquicos de la pirámide de automatización bajo un entorno de Industria 4.0. En la Figura 10 se observa los 5 niveles de la pirámide, cada uno con sus respectivos elementos, además, en los diferentes niveles se muestran las tecnologías que integran la Industria 4.0 como por ejemplo CyberVision (visión cibernética), Cisco ISE (Cisco motor de servicios de identidad), Google Cloud (plataforma en la nube de google), Remote Access (acceso remoto), Wireless Sensor Network (red de sensores inalámbricos), Apps (aplicación móvil); redes de comunicación Ethernet, ProfiNET, entre otras.

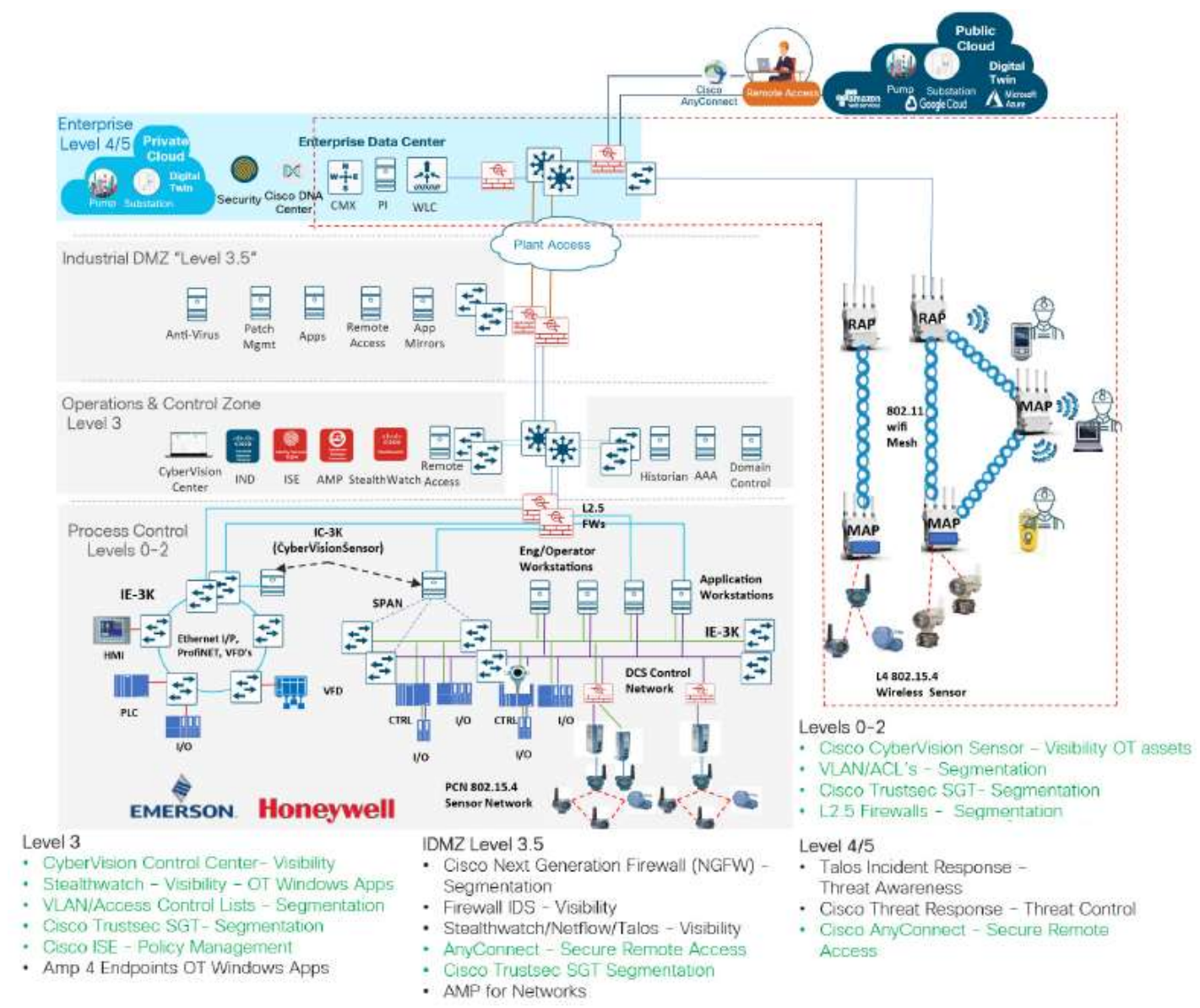

Figura 10. Arquitectura de automatización de un proceso de refinación de petróleo con tecnología de Industria 4.0 (CISCO, 2020)

\section{Análisis de resultados}

En la evaluación de la arquitectura de automatización de los procesos industriales se describieron los instrumentos de campo, control y sistema SCADA, en relación a la pirámide de automatización.

La teoría de Industria 4.0 que fue estructurada en relación a la pirámide de automatización establece una relación con la arquitectura de automatización de los procesos industriales presentados. 
La automatización de los procesos industriales muestra que en la actualidad se está combinando la tecnología de buses de campo con la tecnología de redes Wireless para la manufactura de productos.

A partir de la pirámide de automatización fueron descritos los niveles de automatización de varios procesos industriales, los cuales realizan su función mediante la transferencia de datos por redes de comunicación industrial como PROFIBUS, PROFINET, Ethernet, HART, WirelessHART, redes que son utilizadas en la actualidad por la Industria 4.0.

\section{Conclusiones}

- La red de comunicación Ethernet por su alta capacidad de transmitir datos entre los niveles de empresa, cableado de gran extensión, facilidad de comunicación entre controladores e ideal para redes de área local (LAN), es utilizada en el Nivel 2, Nivel 3 y Nivel 4, mientras que las redes de comunicación PROFIBUS, PROFINET, HART, IO-Link y AS-Interface, por su transferencia de datos en tiempo real entre instrumentos de campo y controladores, trabajo en ambientes explosivos, manejo de tráfico de datos, recuperación de datos en la red, entre otras, es utilizada en el Nivel 0 y Nivel 1 en la automatización de procesos industriales.

- La industria 4.0 marca una gran diferencia con los sistemas de automatización centralizados, ya que al utilizar tecnología de buses de campo y/o tecnología de redes Wireless, se crean sistemas de automatización descentralizados.

- La automatización industrial mediante tecnología de red Wireless va a ser implementada en gran parte de los procesos de manufactura ya que es parte de los conceptos de fábrica flexible en la industria 4.0.

\section{Referencias bibliográficas}

ARC Advisory Group. (2015). How Profinet and Industrie 4.0 Enable InformationDriven Industries. USA: ARC Advisory Group. Recuperado de https://www.profibus.com/download/arc-industrie-40-white-paper

Barrientos, J., \& Gambao, E. (2014). Sistemas de Producción Automatizados. España: Dextra Editorial S.L.

Caler, D. (2015). Análisis y estudio de comunicaciones industriales para implementar arquitectura de comunicaciones estándar en Planta Estándar de Ciclo Combinado (Tesis de Maestría). UAH. Alcalá de Henares

CISCO. (2020). Industrial Automation for Process Control and Refineries Design Guide. Cisco Systems, Inc.

García, E. (2001). Automatización de procesos industriales. España: U.P.V. 
Higuera, A., \& Castillo, F. (2007). CIM: EL COMPUTADOR EN LA AUTOMATIZACIÓN DE LA PRODUCCIÓN. España: Ediciones de la Universidad de Castilla-La Mancha

Hurtado, J. (2015). Introducción a las Redes de Comunicación Industrial. España. Recuperado de https://www.lawebdelprogramador.com/pdf/15495Introduccion-a-las-Redes-de-Comunicacion-Industrial.html

Lozano, M., \& Zamora, R. (2008). Tecnologías y herramientas de ingeniería asociadas a los niveles superiores de la pirámide de la automatización (Tesis de Ingeniería). Universidad Tecnológica de Bolívar. Cartagena de Indias.

Pérez, E. (2015). Los sistemas SCADA en la automatización industrial. Revista Tecnología en Marcha, 28(4), 3-14.

Prado, J. (2010). ETHERNET INDUSTRIAL: Modelos y conectividad en el ámbito de procesos industriales (Tesis de Maestría). Universidad Nacional de La Plata. Argentina.

Profibus. (2019). PROFINET y PROFIBUS PA proporcionan conjuntamente el mejor valor en el control de la planta de embotellado. Recuperado de https://www.profibus.com/technology/case-studies/bestvalue-in-bottlingplant

Salazar, C., \& Correa, L. (2011). Buses de campo y protocolos en redes industriales. Ventana informática, (25), 83-109.

Salvendy, G. (2001). HANDBOOK OF INDUSTRIAL ENGINEERING Technology and Operations Management. USA: John Wiley \& Sons.

Siemens. (2016). Instrumentación de campo para la automatización de procesos. Alemania: Siemens AG. Recuperado de https://support.industry.siemens.com/cs/document/109069191/archivo\%3 A-fi-01-cat\%C3\%A1logos-instrumentaci\%C3\%B3n-de-campo-paralaautomatizaci\%C3\%B3n-de-procesos?dti=0\&lc=es-PA

Siemens. (2021). Productos para la instrumentación de procesos (Catálogo FI 01-2021). Alemania: Siemens AG. Recuperado de https://support.industry.siemens.com/cs/document/109745616/cat\%C3\%A 1logo-fi-01-\%C2\%B7-2021-productos-para-la-instrumentaci\%C3\%B3nde-procesos?dti $=0 \& 1 \mathrm{c}=\mathrm{es}-\mathrm{WW}$

Tecnológico de Monterrey. (2015). Industria 4.0: fundamentos y sus alcances en el sistema eléctrico. Recuperado de https://repositorio.tec.mx/bitstream/handle/11285/636029/20.pdf?sequenc $\mathrm{e}=1$ \&isAllowed $=\mathrm{y}$ 
Universidad de Valencia. (2009). Tema 3. Redes de comunicación industriales. Recuperado de https://www.uv.es/rosado/courses/sid/sid.html

Witorg, (2019). Pirámide de la automatización e industria 4.0. Recuperado de https://www.witorg.org/piramidede-la-automatizacion-e-industria-4-0/

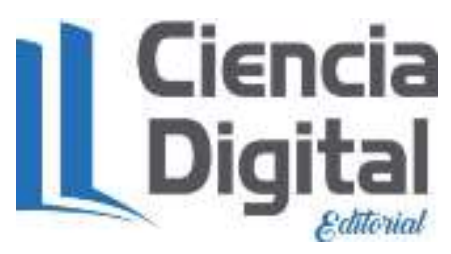




\section{PARA CITAR EL ARTÍCULO INDEXADO.}

Barona López, G., \& Luis Efraín Velasteguí. (2021). Automatización de procesos industriales mediante Industria 4.0 . AlfaPublicaciones, 3(3.1), 98-115. https://doi.org/10.33262/ap.v3i3.1.80

\section{Ligital}

El artículo que se publica es de exclusiva responsabilidad de los autores y no necesariamente reflejan el pensamiento de la Revista Alfa Publicaciones.

El artículo queda en propiedad de la revista y, por tanto, su publicación parcial y/o total en otro medio tiene que ser autorizado por el director de la Revista Alfa Publicaciones.
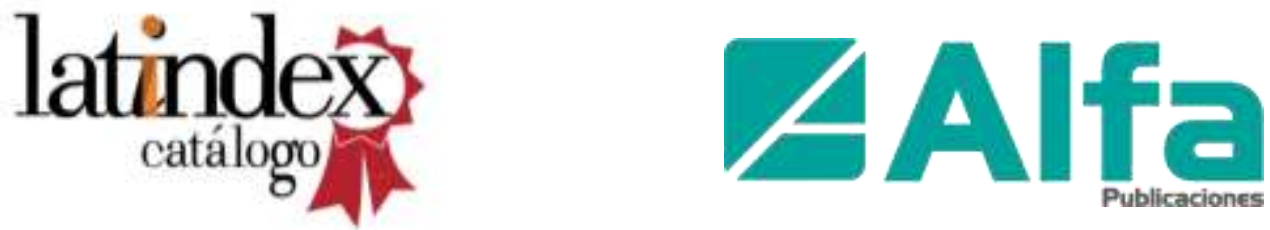